\title{
Diagnostic values of DAS28 and DAS28-squeeze in evaluating Rheumatoid Arthritis disease
}

\author{
David Santosa ${ }^{1}$, Rudy Hidayat ${ }^{2}$, Marcel Prasetyo ${ }^{3}$, Pringgodigdo Nugroho ${ }^{4}$
}

\begin{abstract}
${ }^{1}$ Internal Medicine Departement, Medical Faculty of University of Indonesia, Jakarta ${ }^{2}$ Rheumatology Division, Internal Medicine Department, Medical Faculty of University of Indonesia, Jakarta ${ }^{3}$ Radiology Department, Medical Faculty of University of Indonesia Jakarta

${ }^{4}$ Internal Medicine Department / Clinical Epidemiology Unit, Medical Faculty of University of Indonesia, Jakarta
\end{abstract}

Corespondence: David Santosa, MD $71^{\text {st }}$ Diponegoro Street Central Jakarta, Zip Code 10430

Email :

dr.davidsantosa@ gmail.com

\begin{abstract}
Background : In recent years, rheumatoid arthritis (RA) uses a "treat to target" treatment strategy. This strategy requires a valid and accurate tool for assessing disease activity. The most widely used tool is DAS28, which was developed from DAS with the omission of ankle and foot joints. There has been many critization about the accuracy of DAS28 in classifying the state of RA disease. Most importantly, when an active disease state was misclassified as an inactive state (false negative) which lead to under treat and subsequently to disability. The difference between DAS28 and DAS lies mainly in the exclusion of ankle and foot joints, thus DAS28squeeze, a new and simple tool has been proposed. It comprises the same 28 joints in DAS28 added with a sqeeze test on both metatarsophalangeal joints. However, this new tool has never been validated with any imaging techniques.
\end{abstract}

Objective : To assess the diagnostic values of DAS28 and DAS28-squeeze.

Methods : This study comprised a cross-sectional diagnostic study, using Power Doppler sonography as a standard reference in evaluating the diagnostic value of DAS28 and DAS28-squeeze. This study uses the most sringent sonography criteria of active disease which is an active Doppler signal with a moderate synovial hypertrophy on B-mode.

Results : Over the study period, 56 subjects underwent diagnostic tests using DAS28, DAS28-squeeze and Power Doppler sonography. There were 4 false negative cases in DAS28 and 1 case in DAS28-squeeze. The sensitivities of DAS28 and DAS28-squeeze to identify active disease using Power Doppler sonography as reference standard were $73.3 \%(95 \% \mathrm{Cl} \pm 11.59)$ and $93.3 \%(95 \% \mathrm{Cl} \pm 6.55)$, respectively. While the specificities of DAS28 and DAS28-squeeze were $36.6 \%(95 \% \mathrm{Cl} \pm 12.62)$ and $34.1 \%(95 \% \mathrm{Cl} \pm 12.42)$, respectively. Furthermore the negative likelihood ratio of DAS28 and DAS28-squeeze were 0.73 and 0.19 , respectively.

Conclusion : This study is the first to validate DAS28squeeze using imaging techniques. From this study the false negative rate of DAS28-squeeze is lower than DAS28. DAS28-squeeze has a better sensitivity and negative likelihood ratio than DAS28 in identifying RA disease state.

Keywords : Rheumatoid Arthritis, DAS28, DAS28squeeze, treat to target, Power Doppler, squeeze test

\section{Introduction}

In the past two decades, there has been significant advancement in the understanding of Rheumatoid Arthritis (RA) pathophysiology and therapeutic modalities. Therefore, the need of early detection, early therapy initiation, and tight control of disease activity have risen. Recently, rheumatologists adopted the "treat to target" therapeutical protocol for treating early RA. ${ }^{1-4}$ The main strategy of the therapy is assessing disease activity on a regular basis to achieve a certain target. In daily practice disease activity assessment is usually done with Disease Activity Score 28 (DAS28) because it is clinically applicable. ${ }^{1-3}$ DAS28 has been developed and validated using data from clinical research. Unfortunately, a valid instrument developed from a set of clinical research data is not neccesary became a valid instrument in daily practice. ${ }^{5}$ Thus, a new instrument called DAS28-squeeze developed to assess RA disease activity in clinical practice. This new instrument has never been validated by imaging techniques. Thus, we attempt to assess the diagnostic values of DAS28 and DAS28-squeeze using power doppler sonography as a standard reference.

DAS28 was originally developed from Disease Activity Score (DAS) which was published by van der Heijde, et al. in 1990. The original DAS comprises of 4 components, a 44 swollen joint count, Ritchie articular index (RAI), erythrocyte sedimentation rate (ESR) and patient global assesment of disease. ${ }^{6}$ Because this instrument isn't practically used in clinical settings, in 1994, Prevoo, et al. proposed a modified DAS which comprises only 28 swollen and tender joint count, and excludes the feet and RAI. The modified DAS is now known as DAS28. ${ }^{7}$ DAS28 formula is presented below. ${ }^{1}$

DAS28 $=0.56 \times \sqrt{ }(28 \mathrm{TJC})+\mathbf{0 . 2 8} \times \sqrt{ }(\mathbf{2 8 S J C})$ $+0.70 \times \operatorname{Ln}(\mathrm{ESR} / \mathrm{CRP})+0.014 \times \mathrm{VAS}$

Abbreviation list:

- TJC = Tender Joint Count (jumlah sendi yang nyeri)

- SJC = Swollen Joint Count (jumlah sendi yang bengkak)

- ESR = Erythrocyte Sedimentation Rate (Laju Endap Darah [LED])

- $\mathrm{CRP}=C$-reactive protein

- VAS = Visual Analogue Scale 
DAS28 calculation then can be classified as follows:

- Remission : DAS28 $\leq 2.6$

- Low disease activity : $2.6<$ DAS $28 \leq 3.2$

- Moderate disease activity : $3.2<$ DAS2 285.1

- High disease activity : DAS28 $>5.1$

DAS28 comprises 28-joint, by excluding joints of the feet and ankle. The shortage of DAS28 lies when assessing patient who suffers of inflamation only in his/her feet and ankle joint. DAS28 will classified such a patient as in remission state (inactive disease activity). This could lead to a false negative misclassification, where an active disease activity classified as in a remission state. ${ }^{1,5,6}$ Literatures stated that up to $40 \%$ patients classified as remission by DAS28 still have feet complaints (swollen and/or tender on at least one metatarsophalangeal [MTP] joint). ${ }^{1,8,10,11}$ Further, remission patients, according to DAS28, were also found to have a significant swollen joint counts. $1,8,10,11$

Feet complaints as an initial symptoms has been found in $60 \%$ of RA patients, and after 2 years the feet involvement prevalence decreases to $36 \%$. This feet involvement is an important factor to cause disability in subsequent RA disease proggression. ${ }^{1,7-9}$

Assessing MTP joints inflamation (synovitis) in daily clinical settings is cumbersome. Thus, a simple test has been proposed to assess synovitis in feet joints to improve disease activity measurement, which in turn will influence the subsequent therapeutic decision making. This test is the MTP joints bilateral squeeze test which assess the bilateral compression pain (BCP). ${ }^{1,10}$

DAS28 which modified by adding MTP squeeze test is called DAS28-squeeze. It is developed using linear regression model using DAS as a dependent variable and DAS28 and MRP squeeze test as an independent variables. The squeeze test results are categorized as follows: 0 for negative test (no pain on both feet), 1 for pain on one foot, 2 for pain on both feet. De Jong, et al. defined the disease activity state for DAS28-squeeze as follows: below 1.6 for remission, 1.6 - 2.4 for moderate disease activity and above 2.4 for high disease activity. Whereas the DAS28-squeeze formula is $0.64 \mathrm{x}$ DAS28 $+0.23 \times$ squeeze test result. This new instrument has also never been validated by imaging techniques. de Jong, et al. stated that imaging validation is required to assess DAS28squeeze validity. ${ }^{1}$

Up till now, RA disease activity assessment in clinical settings has relied on DAS28. Disease activity assessment is an important aspect of RA management because theraupetical decision is given based on the disease activity state. If DAS28 is inaccurate to classify RA disease activity, patients would get suboptimal therapy which leads to rapid disease progression and ultimately to disability. In this study, we would like to assess the diagnostic values of DAS28 and DAS28-squeeze comparing with power doppler sonography as a reference standard.

\section{Methods}

This study is a diagnostic study using power doppler sonography as reference standar. Study subjects are patients visiting Cipto Mangunkusumo hospital Rheumatology outpatient clinic between August to December 2015 period who fulfilled the $2010 \mathrm{ACR} / \mathrm{EULAR}$ criteria for rheumatoid arthritis and who were willing to be tested using power doppler sonography and didn't suffer from any infection (tuberculosis, pneumonia, urinary tract infection, other bacterial infection) that might influence their ESR result. Calculated sample size were 47 patients, this was achieved using the following formula.

$$
\mathrm{n}=(\mathrm{Z} \alpha)^{2} \frac{\operatorname{Sen}(1-\operatorname{Sen})}{\mathrm{d}^{2} \mathrm{P}}
$$

Sensitivity was set at $90 \%$, study precision (d) was set at $10 \%$, $\mathrm{Z} \alpha$ was set at 1.96 and prevalence (P) of active disease was set at $75 \%$.

Sampling was done using consecutive sampling method. Diagnostic values of DAS28 and DAS28-squeeze were stated as sensitivity, specificity, positive predictive value, negative predictive value, positive likelihood ratio and negative likelihood ratio using $95 \%$ conficence interval. All calculations were done using Statistical Packages for Social Sciences (SPSS) version 20.

Squeeze test was done by one single examiner on both metatarsophalangeal (MTP) joints by placing thumb and index finger, respectively, just below the first MTP joint and over the fifth MTP joint. The thumb is placed just below the first MTP joint to prevent direct compression of the joint. Then, the metatarsal joints are bilaterally compressed, using a force equal to handshake. The squeeze test was coded as follows: 0 $=$ test negative on both forefeet, $1=$ test positive on 1 side, $2=$ test positive on both forefeet. ${ }^{1}$

Power doppler ultrasonography (PDUS) was done by one single radiologist experienced in musculoskeletal ultrasonography. The radiologist was blind about the result of DAS28 and MTP squeeze of each subject. PDUS were used to examine joints as stated in US7 joints, include wrist, second and third metacarpophalangeal and proximal interphalangeal, and second and fifth metatarsophalangeal joints. ${ }^{11}$ Grey-scale imaging evaluation confirmed the presence or absence of synovial hypertrophy ( $\mathrm{SH}$ ) and/or joint effusion, which was graded using a semiquantitative scoring method consisting of a scale of $0-3$, where 0 represented no SH, 1 mild hypertrophy, 2 moderate hypertrophy and 3 severe hypertrophy. PD was stated as 0 represented no signal and 1 as positive power doppler signal. ${ }^{12}$ Synovitis on PDUS was defined using the most stringent PDUS criteria which is $\mathrm{SH} \geq 2$ with positive doppler signal. ${ }^{12,13}$

\section{Results}

Fifty six subject (49 females and 7 males) were included in this study. Study subject characteristic can be seen in Table 1 . 
Table 1. Study subject characteristic

\begin{tabular}{lll}
\hline Subject characteristic & N & $\%$ \\
\hline 1. Sex & & \\
Male & 7 & 12.5 \\
Female & 49 & 87.5 \\
2. Age Group & & \\
21-30 years old & 3 & 0.05 \\
31-40 years old & 10 & 17.86 \\
41-50 years old & 11 & 19.65 \\
51-60 years old & 17 & 30.36 \\
> 60 years old & 15 & 26.78 \\
3. Disease duration & & \\
$\quad$-2 years & 20 & 35.72 \\
$\quad>2$ years & 36 & 64.28 \\
4. Feet complaints & & \\
Present & 25 & 44.64 \\
None & 31 & 55.36 \\
6. Medication & & \\
MTX & 32 & 57.14 \\
SFZ & 3 & 0.05 \\
MTX + SFZ & 5 & 0.09 \\
MTX + MP & 13 & 23.21 \\
SFZ + MP & 2 & 0.03 \\
MTX + SFX + MP & 1 & 0.01 \\
5. Body Mass Index & & \\
Underweight & 31 & 0.05 \\
Normal & 22 & 55.35 \\
Overweight & & \\
\hline
\end{tabular}

MTX = Methotrexate, SFZ = Sulfasalazine, MP = Metilprednisolon

Study subject were predominantly women $(87.5 \%)$, most were between 51-60 years old (30.36\%), with mean age of 50.6 years old. Most of the patients $(64.28 \%)$ who visited Cipto Mangunkusumo hospital Rheumatology outpatient clinic suffers from chronic RA, with mean disease duration of 4.8 years. Twenty five $(44.64 \%)$ patients had feet complaints.

\section{DAS28 and DAS28-squeeze}

Below are presented the result of DAS28 and DAS28-squeeze test.

Table 2. DAS28 and DAS28-squeeze results

\begin{tabular}{lll}
\hline Instrument & $\mathbf{n}$ & $\%$ \\
\hline DAS28 & & \\
$\quad$ Inactive & 19 & 33.93 \\
$\quad$ Active & 37 & 66.07 \\
DAS28-squeeze & & \\
$\quad$ Inactive & 15 & 26.78 \\
$\quad$ Active & 41 & 73.22 \\
\hline
\end{tabular}

DAS $=$ Disease Activity Score

\section{Kappa score for sonography test}

To assess the reliability of sonography examination, we decided to repeat the sonography examination on $10 \%$ of the total estimated sample, resulted in five subjects on all seven joints included in the examination with totally 35 joints. The first and second sonography examination was done on the same day to avoid any changes on the synovitis activity. All sonography examinations were done by the same examiner.
From the examinations a $2 \times 2$ table could be constructed as follows.

Table 3 Sonography Kappa test

\begin{tabular}{llccc}
\hline positive & & \multicolumn{2}{c}{ First Test } & \multirow{2}{*}{ total } \\
\cline { 2 - 4 } & & negative & \\
\hline \multirow{2}{*}{$\begin{array}{l}\text { Second } \\
\text { Test }\end{array}$} & Positive & 17 & 3 & 20 \\
\cline { 2 - 5 } & Negative & 4 & 11 & 15 \\
\hline & Total & 21 & 14 & 35 \\
\hline
\end{tabular}

The Kappa score result according to SPSS version 20 was 0.588 which reflected a moderate to good reliability.

\section{Diagnostic Test Results}

From the study data we could construct $2 \times 2$ tables of DAS28 and DAS 28-squeeze, as follow.

Table 4. DAS28 diagnostic test

\begin{tabular}{|c|c|c|c|c|}
\hline \multirow[t]{2}{*}{ Active } & & \multicolumn{2}{|c|}{ USG } & \multirow[t]{2}{*}{$\mathrm{n}$} \\
\hline & & Inactiv & & \\
\hline \multirow{3}{*}{ DAS28 } & Active & 11 & 26 & 37 \\
\hline & Inactive & 4 & 15 & 19 \\
\hline & & 15 & 41 & 56 \\
\hline
\end{tabular}

Table 5. DAS28-squeeze diagnostic test

\begin{tabular}{|c|c|c|c|c|}
\hline \multirow[t]{2}{*}{ Active } & & \multicolumn{2}{|c|}{ USG } & \multirow[t]{2}{*}{$\mathrm{n}$} \\
\hline & \multicolumn{3}{|c|}{ Inactive } & \\
\hline DAS28- & Active & 14 & 27 & 41 \\
\hline squeeze & Inactive & 1 & 14 & 15 \\
\hline \multicolumn{2}{|c|}{ Total } & 15 & 41 & 56 \\
\hline
\end{tabular}

Based on the tables we could calculate DAS28 diagnostic values as follows; sensitivity $73.3 \% \%(95 \% \mathrm{CI} \pm 11.59)$, specificity $36.6 \%(95 \% \mathrm{CI} \pm 12.62)$, positive predictive value $29.7 \%(95 \% \mathrm{CI} \pm 11.97)$, negative predictive value $78.9 \%$ $(95 \% \mathrm{CI} \pm 10.69)$, positive likelihood ratio 1.15 and negative likelihood ratio 0.73 . And DAS28-squeeze diagnostic values; sensitivity $93.3 \%(95 \% \mathrm{CI} \pm 6.55)$, specificity $34.1 \%(95 \% \mathrm{CI}$ $\pm 12.42)$, positive predictive value $34.1 \%(95 \% \mathrm{CI} \pm 12.42)$, negative predictive value $93.3 \%(95 \% \mathrm{CI} \pm 6.55)$, positive likelihood ratio 1.41 and negative likelihood ratio 0.19 .

\section{Discussion}

Synovial hypertrophy on B-mode sonography was found in $91.07 \%$ of wrist joints examinations, this finding is consistent with Doron et al study which concluded around $75 \%$ of early RA patients have wrist complaints and could increase to up to $95 \%$ in chronic $\mathrm{RA}^{14}$ Vlad, et al. study concluded that power doppler sonography is the best modality in assessing pathological activity in wrist and finger joints. They also concluded that wrist joint is the most prevalently affected joint in RA. ${ }^{14}$

The low specificity result in this study could attribute to the high false positive number in both DAS28 and DAS28- 
squeeze. Which means many patients that were in an active disease state according to DAS28 and DAS28-squeeze were found to be inactive according to sonography examination. There are a few possible explanations for this high number of false positive. First, many patients that have swollen and tender joints were found to be inactive according to sonography. This is possible because most patients included in this study are suffered from chronic RA which resulted in synovial thickening, thus making it more difficult to detect blood flow on power doppler sonography. Other explanation is that many patients have already developed joint deformity which resulted in pain on joint counts, but not neccesarily reflects the active inflammation state. ${ }^{15}$ Second, the formula used in DAS28 emphasizes too much on ESR, thus little increase in ESR produced a significant increase in DAS28 result. ${ }^{5}$ Since DAS28-squeeze uses DAS28 result in its calculation, the value would also be significantly increased. The third possibility is undetected synovitis by sonography examination. This is caused by several reason, such as: (1) excessive pressure applied on examination area; (2) low skin temperature resulted in decrease in Doppler activity, thus cleaning with water prior to examination should be avoided; (3) lack of gel applied; (4) diurnal variation in Doppler activity; (5) sonography equipment settings. ${ }^{13,}{ }^{16,17}$ However a negative Doppler signal doesn't always reflected an inactive disease state. ${ }^{13}$

In this study all avaoidable factors that could resulted in undetection of Doppler activity have been anticipated. Gel application was adequate, excessive pressure wasn't applied, water wasn't applied prior to examination, equipment settings were good and the operator was experienced in musculoskeletal sonography. This study uses the most stringent sonography criteria in defining active synovitis.

False positive in DAS28 and DAS28-squeeze were found in 28 and 26 cases, respectively. The consequences of the false positivity is an over treatment in treating RA patients. DAS28-squeeze sensitivity of $93.3 \%(95 \% \mathrm{CI} \pm 6.55)$ shows that this instrument is quiet good for screening purpose. A likelihood ratio of 0.19 also indicating that it is good enough to rule out the disease, because ideally it should be below 0.1 . DAS28-squeeze power in ruling out active disease state is far better than DAS28, which can be seen from DAS28 sensitivity and negative likelihood ratio of just $73.3 \%(95 \% \mathrm{CI} \pm 11.59)$ and 0.73 respectively. From the likelihood ratio results we can calculate the post-test probability of each instrument as follows:

Table 6 Post-test probability results

\begin{tabular}{cccccccccc}
\hline & & Positive Post- & Negative & Positive Post- & Negative Post- \\
Pretest & Pre- & \multicolumn{2}{c}{ test Odds } & Post-test Odds & test Probability & test Probability \\
Prob- & test & DAS & DAS 28- & DAS & DAS 28- & DAS & DAS 28- & DAS & DAS 28- \\
ability & Odds & 28 & squeeze & 28 & squeeze & 28 & squeeze & 28 & squeeze \\
\hline $75 \%$ & 3 & 3.45 & 4.23 & 2.19 & 0.57 & $77.5 \%$ & $80.9 \%$ & $68.6 \%$ & $36.3 \%$ \\
\hline
\end{tabular}

False negative were found in 4 cases and 1 case in DAS28 and DAS28-squeeze respectively. False negativity in RA management should be avoided because it could lead to under treatment which could ultimately resulted in disability. This study results are in line with the initial goal of DAS28-squeeze in avoiding false negative misclassification.

\section{Conclusion}

This study is the first study which evaluates diagnostic values of DAS28-squeeze using imaging techniques as reference, particularly using power doppler sonography criteria in detecting active RA disease activity state. The limitation of this study primarily lies in the characteristic of subject where most of them suffer from chronic RA which resulted in harder Doppler activity detection. In conclusion DAS28-squeeze has better diagnostic values than DAS28 in evaluating RA disease activity state using power doppler sonography as reference.

\section{Reference}

1. de Jong $P H$, Weel $A E$, de Man YA, Huisman $A M$, Gerards $A H$, van Krugten MV, et al. To squeeze or not to squeeze, that is the question! Optimizing the disease activity score in 28 joints by adding the squeeze test of metatarsophalangeal joints in early rheumatoid arthritis. Arthritis \& Rheumatism 2012;64(10):3095-3101.

2. Farheen K, Agarwal SK. Assessment of disease activity and treatment outcomes in rheumatoid arthritis. J Manag Care Pharm 2011;17(9-b):S913.

3. Alvarado PM, Laiz A. Is DAS a profitable score to be used for rheumatoid arthritis patient follow up? Reumatol Clin. 2011;7(5):336-338.

4. Chau LS-Y, Mok C-C. Assessment of disease activity and functional outcome in rheumatoid arthritis. Hong Kong Bulletin on Rheumatic Diseases. 2002;2(1):10-14.

5. Gardiner P. Misreading disease activity with DAS28 [Internet]. Norwalk: Rheumatology network; March 17, 2014 [cited 2015]. Available from:www.rheumatologynetwork.com.

6. Landewe R, van der Heijde D, van der Linden S, Boers M. Twenty-eightjoint counts invalidate the DAS28 remission definition owing to the omission of the lower extremity joints: a comparison with the original DAS remission. Ann Rheum Dis 2006;65:637-641.

7. Borman P, Ayhan F, Tuncay F, Sahin M. Foot problems in a group of patients with rheumatoid arthritis: An unmet need for foot care. The Open Rheumatology Journal. 2012;6:290-295.

8. van der Leeden M, Steultjens MPM, Ursum J, Dahmen R, Roorda LD, Schaardenburg Dv, et al. Prevalence and course of forefoot impairments and walking disability in the first eight years of rheumatoid arthritis. Arthritis \& Rheumatism 2008;59(11):1596-1602.

9. Siddle H, Redmond A, Wakefield R, Hodgson R, Grainger A, Pickles D, et al. Prevalence of MTP joint involvement in the painful forefoot of patients with rheumatoid arthritis. Journal of Foot and Ankle Research 2010;3 (Suppl 1):023.

10. Wiesinger T, Smolen JS, Aletaha D, Stamm T. Compression test (Gaenslen's Squeeze Test) positivity, joint tenderness, and disease activity in patients with rheumatoid arthritis. Arthritis Care \& Research 2013;65(4):653-657.

11. Backhaus M, Ohrndorf S, Kellner H, Strunk J, Backhaus T, Hartung W, et al. Evaluation of a novel 7-joint ultrasound score in daily rheumatologic practice: A pilot project. Arthritis \& Rheumatism 2009;61 (9):1194-1201.

12. Balsa A, Miguel Ed, Castillo C, Peiteado D, Martın-Mola E. Superiority of SDAI over DAS-28 in assessment of remission in rheumatoid arthritis patients using power Doppler ultrasonography as a gold standard. Rheumatology 2010;49:683-690.

13. Koski J, Saarakkala S, Helle M, Hakulinen U, Heikkinen J, Hermunen H. Power Doppler ultrasonography and synovitis: correlating ultrasound imaging with histopathological findings and evaluating the performance of ultrasound equipments. Ann Rheum Dis 2006;65:1590-1595. 
14. Doron II, Michael ER. Rheumatoid Arthritis of the Wrist. Hospital for Joint Diseases 2003-2004;61 (3 \& 4):179-185.

15. Elfattach H, Houari F, Addou O, Maaroufi M, Tizniti S. Sonographic appearance of chronic inflammatory rheumatism [Poster in ECR 2013 Congress]. European Society of Radiology; 2014. DOI: 10.1594/ ecr2013/C-2237
16. Filippucci E, Salaffi F, Carotti M, Grassi W. Doppler ultrasound imaging techniques for assessment of synovial inflammation. Reports in Medical Imaging 2013;6:83-91.

17. Semerano L, Gutierrez M, Falgarone G, Filippucci E, Guillot X, Boissier $\mathrm{M}-\mathrm{C}$, et al. Diurnal variation of power Doppler in metacarpophalangeal joints of patients with rheumatoid arthritis: a preliminary study. Ann Rheum Dis 2011;70(9): 1699-1700. 